$\mathbb{T}$ periodica polytechnica

Transportation Engineering

$37 / 1-2$ (2009) $71+75$

doi: 10.3311/pp.tr.2009-1-2.12

web: http://www.pp.bme.hu/tr

(c) Periodica Polytechnica 2009

RESEARCH ARTICLE

\section{Analysis of the COMGUARD advance signalising system for dangerous road traffic points}

\author{
Tamás Berta
}

Received 2008-11-03

\begin{abstract}
The COMGUARD is the advance warning system of hazardous road spots, which consists of signallers built into the road surface, and of onboard unit to be installed into the vehicle. The system elaborated on the basis of an internationally patented innovation was set up with experimental character in 9 counties in Hungary, creating this way a test reference area. On the basis of this, KTI carried out tests and surveys in 20072008 on the compliance and efficiency of the system, analysing the users' attitude and the probable acceptance by the population. In the survey, the attitude of the drivers has been studied in the case of not using and using the device; we evaluated the reliability of the signaller and signalling; we analysed the demands and the acceptance to be expected from the side of the population.
\end{abstract}

\section{Keywords \\ dangerous roads $\cdot$ road safety}

Tamás Berta

Institute for Transport Sciences Non-Profit Ltd, Road Safety and Traffic Engineering Division,, Bp. 1119. Thán Károly u.3-5., Hungary

e-mail: berta.tamas@kti.hu

\section{Introduction}

The system used for the advance signalisation of dangerous road traffic points, called COMGUARD, the result of a 13-year development process, is an internationally registered, patented invention. Essentially, the system means the installation of magnets into the road surface in dangerous road traffic points, and according to the information provided by the magnetic field, a warning is displayed for the driver of the vehicle equipped with COMGUARD sensors. The signalling apparatus of the system created on the basis of technical innovation is practically maintenance-free and it requires no (energy) supply, it cannot be stolen and damaged. It has become the COMGUARD system's current basic principle that it can repeat only regular road signals, signs of the Highway Code.

Beside technical development, though with smaller-greater impetus, some traffic engineering and scientific road research analyses on the device have also been carried out. The experts are on different opinion as to the real road safety returns of such a device and whether the signaller operates reliably in traffic circumstances as well.

Supported by the results of preceding analyses, the implementation of a major survey became possible in 2008 , since the patentee - from his own resources - installed the magnetic sensors in nine counties (in the Trans-Danubian region, except Pest County). The article presents the circumstances and the results of the analysis.

\section{The technical set-up of the COMGUARD system}

The COMGUARD multi-functional information system consists of two completely separate main units: on the so-called primary side of the magnetic signaller, and on the secondary side (in the vehicle) of the sensor, as well as of the display units.

Magnetic signaller: the signalling magnets built into the road surface function as passive elements. Physically, the signaller means that a coded, permanent magnetic field can be created by the spacing and the number of permanent magnets with permanent magnetic field, aligned in one or several lines, imbedded into the road surface.

According to the principle of flux density (see Fig. 1), the 


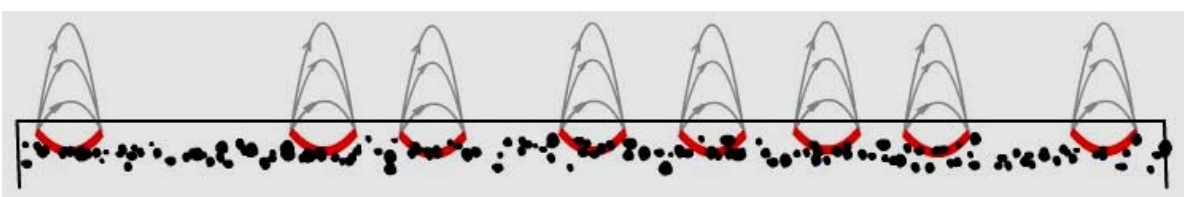

Fig. 1. The signalling magnetic field (schematic diagram)

magnetic field above the surface of the pavement located in a coded way enables the sensor installed in the vehicle to "read" these codes. This way, it is possible to code numerous permanent information data, e.g. railway crossings, interchanges, etc. Appropriate to the risk level of the place, drivers of vehicles equipped with receiving relays, passing through these magnetic fields are always warned visually and acoustically.

Sensor - and display: When passing above the coded magnetic field, the sensor by the induction coil, through coder electronics, transmits a digital sign to evaluating electronics, which controls the central displaying unit.

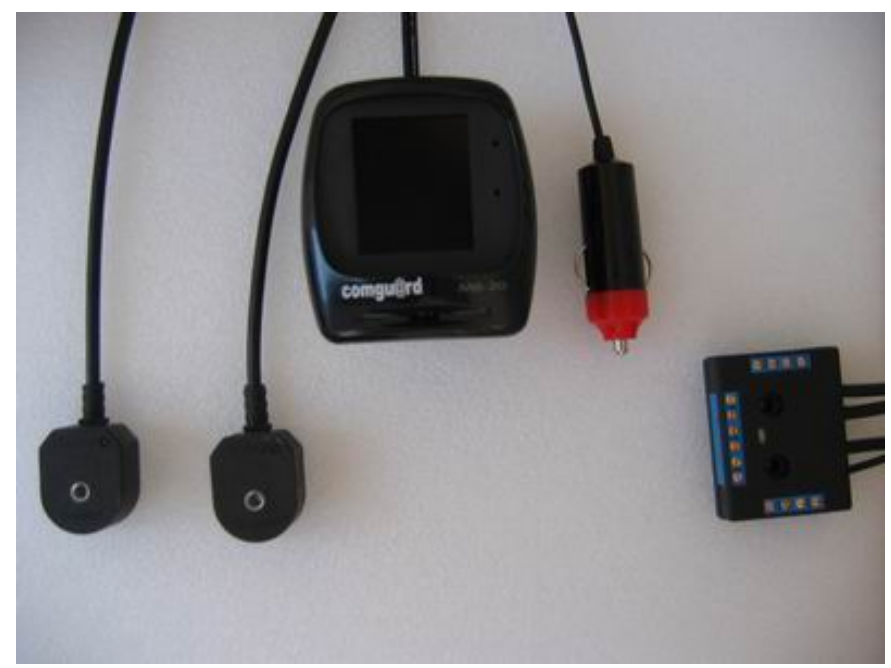

Fig. 2. Display and sensors

Installation in the vehicle of the sensor and the display unit is very simple, it needs no expertise. The sensor placed on the floor in front of, or below the front seats of the vehicle is ready for operation. The displaying unit can be easily fixed to the meeting point of column 'A' on the left side and the instrument board (it is not disturbing, not covering the field of vision) (Fig. 2).

\section{Course of the measurement-test}

Measurement-tests have been carried out, and the experiences of the users of the device have been collected in order to analyse the efficiency, the operation and the reliability of the COMGUARD system, and its effect on drivers.

Test I - reliable registration of the signal: One of the most important parts of the test was its Transdanubian deployment: the survey of this reference system, with the primary aim to evaluate and test the signalling fields, the so-called primary side. The main area of the measurement-tests is the technical inspection and compliance analysis of the reference system. To this aim, a measuring system has been developed appropriate to test the
COMGUARD in traffic conditions (so-called floating car). The measuring system registers and stores the travel dynamics of the vehicle, if it passes over the COMGUARD field, consequently the acceleration data, the speed, the positions, as well as the signal's obtained picture will also be known. The measurement results were recorded in every two minute intervals on the different sites, thus the data preceding and following the signal could be compared. Software has been prepared for evaluation, which is also appropriate for the signalling fields (Fig. 3).

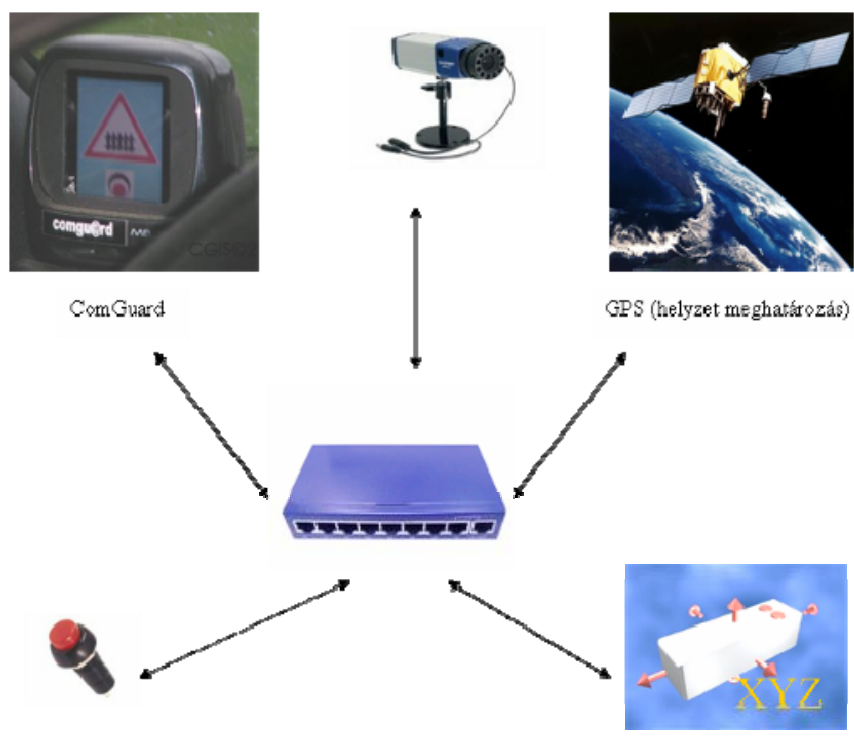

Fig. 3. Schematic diagram and active picture of evaluation software of the measuring system

The software has been used to check the reliability of the information gathered in respect of all records. It has been checked whether the obtained signal is really the planned information and the COMGUARD device "brought forward" the signal according to the field.

Test II - Inspection of the effect influencing the drivers' behaviour - observation, recording of reactions: The test consists of two parts, a daytime and a night-time measurement; its aim is to study the driving behaviour of different drivers, with and without the COMGUARD system, in appropriate and limited (nighttime) visibility conditions. Ten drivers drove (several times) along a longer route with many such risks, which were signalled by the COMGUARD and where the signalling magnetic-fields are installed. Drivers' attitude was recorded by camera(s), the travel dynamics of the vehicle by microcomputer.

Test III - Survey of the demand of the population: Surveying the population in respect of road safety problems, with a random stratified selection of 1500 persons, holders of driving per- 
mits were selected at random and inquired [2]. Most questions can be related indirectly to the concrete system tested (COMGUARD), as the service is not completed yet and the extent of the relevant information is still inadequate for making public surveys. We took stock of the interviewees' general road safety attitude; judged the utility of safety increasing devices and activities; which traffic situations and road traffic sites are considered as the most dangerous ones; after the presentation of other equipment (mainly the GPS based navigation) we surveyed their opinions on the COMGUARD device.

Test IV - Collection of experiences gained by the users of the device: To enable the collection of more plentiful and thorough information, the method of 'controlled interviewing' has been chosen for making the survey. The method is not limited to anticipated replies, and it helps responders (interviewees) to present their experiences and opinions in their own words. As the sample size is small, the use of the COMGUARD device was the only criterion of involvement into the sample.

\section{Probable results of the tests}

Analysing the reliability of signalling, during the totally completed 1322 measurements related to all valid CONGUARD signals, the proportion of false signals was $3 \%$ (in every case this meant that the device gave a signal on a place, where there was no signaller) (Fig. 4).

Under the influence of the device, most participants selected the appropriate speed, however there were also cases, when despite confirmation, the road sign was ignored. Nevertheless, the attentive attitude of drivers was almost entirely positively affected by the device - a fact that is also reflected by travel dynamics. It is interesting that the drivers are preparing with emphasised attention to solve the given traffic situation, manoeuvre, and as a consequence, they gradually moderate their speed - thus, the speed values are becoming more even in time and the acceleration rate more homogeneous. This is definitely a favourable result, because the other road users are also beneficiaries, if in the given case, the attitude of the road participant just passing in front of them is more foreseeable.

Drivers' attitude has perceivably changed under the effect of the signals produced by the COMGUARD system. The head movements of the surveyed participants became slightly more intensive, interventions related to speed reduction became more frequent - it is especially fortunate that this has often been achieved by slight deceleration (engine brake) started from the appearance of the signal, and the number of situations when the driver showed no reactions at all to the changing traffic circumstances decreased by $50 \%$ [4].

During the test, the attitude of the drivers was not only observed in locations signed with advance signals, but also in curves and road bends which - in our opinion - were equally sharp, difficult to solve, but not signed by separate road sign. We would have liked to see whether the drivers' reactions and manoeuvres are different from the attitudes experienced on the sites signalled in advance. It is interesting that on the locations, where there were no signs, the drivers had fewer head movements; they did not use their mirrors, paid less attention to their environment. On the locations which were not signed in advance, the brakes were applied more frequently, as the reduction of the speed started later, the engine brake was more rarely used, and speed-distribution was more inhomogeneous (Fig. 5). The number of events is astonishingly high, when the driver does not react to the change of the road alignment either, does not decelerates, his attention is not increased.

On the one hand, the observations indicate that additional information influenced positively the drivers' capacity of accomplishment of the problems; while on the other hand, it is an important warning which draws the attention to the inconsistency of the basic system (road signs - static information system) and basic network. Now, the reactions clearly show that the road signs by themselves, too, influenced (favourably) the drivers, while the COMGUARD increased the positive effect.

According to population's survey, the drivers believe that safety increasing means play a significant role in safe road traffic.

The survey also proved that in the opinion of the road users, only abilities and routine are not enough for safe traffic. For right decisions, the driver needs vehicle signals, because he depends mainly on the information gathered on traffic environment and on the information provided by, and received from the environment.

Much depends on information type and quantity. Likewise, inadequate information, too, could be a risk source - as a disturbing information set (surplus information), or as an unrealised information.

According to the interviewed road users, a sharp curve and a railway-crossing are considered as main risk sources.

On the first place of the scale, developed on the basis of spontaneous declarations (pcs), it stands: "necessity of more increased or more efficient police enforcement on roads". Modernisation of the road network is mentioned in the second place, and the improvement of the traffic morals - that can be interpreted as the synonym for traffic discipline - was also given a "distinguished position" [3, 5].

In respect of the number of the spontaneous declarations, an outstanding emphasis was laid on education, the strict observance of the speed limit; and among the spontaneous replies given to the question, the 'danger warning device in the vehicle', the 'modernisation of the railway-crossings' and the 'signalling device' appear as well.

The replies given to our questions were also analysed with reference to the COMGUARD system, and on the basis of the relationships reflected, in principle such a device, a system warning to dangerous locations has been "built up", which is mostly based on people's demands. This sample system has been compared with the system-plan of the COMGUARD. The "demands" of the population and the characteristics of the 
Fig. 4. Distribution of the incorrect signals

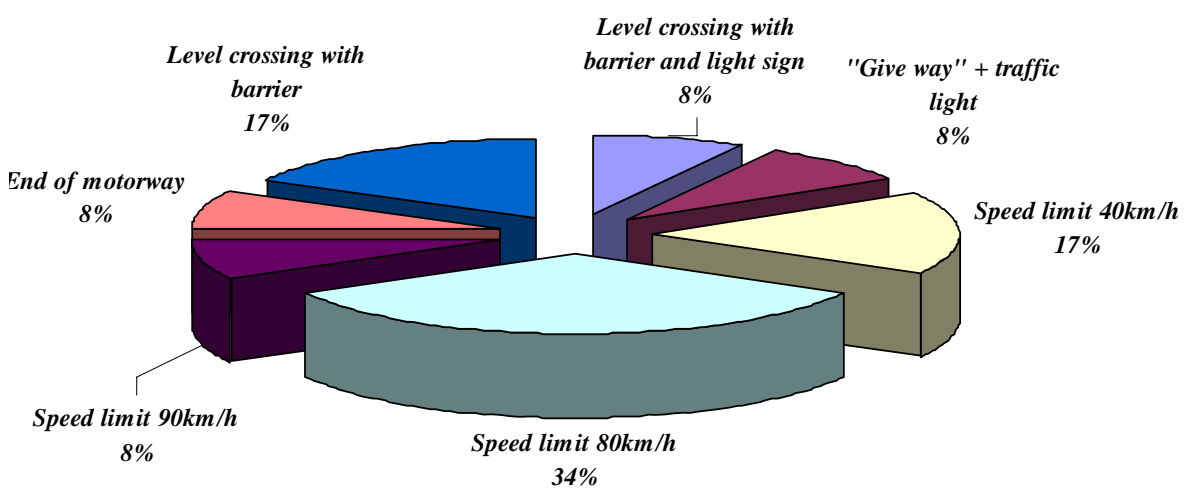

Fig. 5. Driver's behaviour

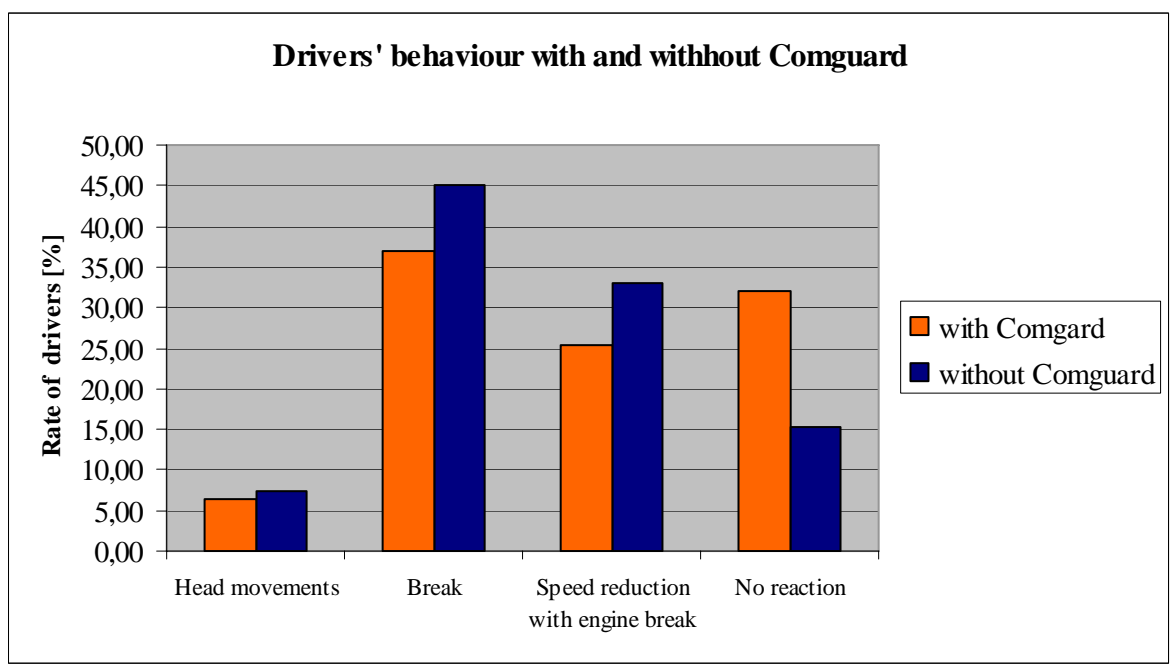

COMGUARD are in line; the population probably will support and accept the system.

The users of the device stressed the necessity of warning to dangerous interchanges. Lack of deployment was always present in the interviews, reflecting also the demand; consequently the countrywide installation of the COMGUARDsystem enjoys a definite support. In addition it has been mentioned that further to the enlargement and updating of the system, care should be taken that only the critical and fix points are worth signalling in advance. Our interviews declared also that Hungarian traffic is overregulated, and this overregulation calls forth the drivers' false feeling of safety. Nevertheless, likewise surplus information, the lack of some important information or its carelessness-related elimination, could also result in accident [6]. Surplus information is not increased by the COMGUARD system, but the most important one is passed to the passenger compartment, confirming the content of the road sign by repeating it.

Summarising the different opinions, the COMGUARD system has been accepted positively; and we can report on positive experiences from the part of connoisseurs and users [1].

\section{Conclusions}

The COMGUARD system is capable to serve safe traffic. It has a positive effect on drivers' attentive attitudes; in certain situations it helps the driver in the safe selection process of the driving speed. The system is technically reliable; the explored mis- takes are not endangering, but they can be eliminated. Basically, the signalling network is free of maintenance; however, permanent monitoring and status-control should be solved. Since actually it repeats the message of the road signs, it "inherits" the basic characteristics of that, together with the mistakes and inconsistencies also. Therefore, it is necessary and indispensable to set these mistakes right before a countrywide installation is implemented. The dangerous sites to be currently signalled are appropriate according to the tests, and in the opinion of the experts, and in that of the drivers and users, as well, doubts worth solving arouse only in respect of speed restrictions. The COMGUARD or some similar equipment can enjoy support if they are appropriately presented to the population. The COMGUARD is capable to satisfy drivers' latent requirements for equipment supporting the road safety with further or better quality information.

The users of the device have a good opinion of the device; they have been motoring since long years with the COMGUARD. All of them support the system and would welcome its improvement.

The interviewed law-expert (also user of the device) finds it useful, too, especially stressing its reason for existence in case of railway crossings (in knowledge of the accident characteristics of these sites).

The COMGUARD may be run as an independent device, forming in itself also a complete system, at the same time its provided service can be joined to other equipment as well (e.g. navigation devices). Other instruments (e.g. mobile phone or 
palmtop) can also be used as alternatives for its onboard display.

Radio communication must have an outstanding role in the improvement process, since it makes also possible the forecasting of suddenly changing, increasingly hazardous circumstances which are causing situations with accident risk. Improvement is running already.

Total, countrywide installation is the requirement of the acceptance of the operation, the running of the system.

Timing of the installation can be envisaged in various ways. The system provides real service only if the different functions are set up countrywide. Therefore, the solution could be that in the first stage the signal of all the railway crossings is installed, then, the service is enlarged within the framework of annual programmes.

It can also be envisaged that unlike the present system, signalling would not be attached to road signs, but to locations with increased accident risk, with limited visibility conditions, etc., which would be marked out by local experts with the involvement of a committee.

The device could be really useful and efficient if it is installed in as many as possible vehicles; its propagation must be an outstanding aim. (According to preliminary calculations the purchasing price of the device can be kept under 10,000 HUF.)

\section{References}

1 KTI - Transport Research Institute Non Profit Ltd., Analysis of the “COMGUARD”tentativesystem, 2005. research documentum.

2 FORSENSE Ltd., Population measuring about Road Safety, 2007.

3 Richard van der Horst R, Kaptein N, Self-Explaining Roads.

4 Purcell A T, Environmental perception and affect an schema discrepancy model, Environment and Behavior (1986).

5 Tánczos K, Török Á, Road safety techniques in Hungary according to EU directives, MOSATT (Modern Safety Technologies in Transportation), 2005, pp. 410-413.

6 Sesztakov V, Török Á, Monetary value of road accidents in Hungary, MOSATT (Modern Safety Technologies in Transportation), 2007, pp. 250253. 